\title{
Evidence of unconventional low-frequency dynamics in the normal phase of $\mathrm{Ba}\left(\mathrm{Fe}_{1-x} \mathrm{Rh}_{x}\right)_{2} \mathrm{As}_{2}$ iron-based supercondutors
}

\author{
L. Bossoni, ${ }_{1}^{1,2}$ P. Carretta, ${ }^{1}$ W. P. Halperin, ${ }^{3}$ S. Oh ${ }^{3}$ A. Reyes,${ }^{4}$ P. Kuhns, ${ }^{4}$ P.C. Canfield ${ }^{5}$. \\ 1 Department of Physics, University of Pavia-CNISM, I-27100 Pavia, Italy \\ 2 Department of Physics "E. Amaldi," University of Roma Tre-CNISM, I-00146 Roma, Italy \\ 3 Department of Physics and Astronomy, NorthWestern University, Evanston, IL, USA \\ ${ }^{4}$ National High Magnetic Field Laboratory, Tallahasse, FL, USA and \\ ${ }^{5}$ Ames Laboratory US DOE and Department of Physics and Astronomy, Iowa State University, Ames, IA 50011, USA
}

\begin{abstract}
This work presents ${ }^{75} \mathrm{As}$ NMR spin echo decay rate $\left(1 / T_{2}\right)$ measurements in $\mathrm{Ba}\left(\mathrm{Fe}_{1-x} \mathrm{Rh}_{x}\right)_{2} \mathrm{As}_{2}$ superconductors, for $0.041 \leq x \leq 0.094$. It is shown that $1 / T_{2}$ increases upon cooling, in the normal phase, suggesting the onset of an unconventional very low-frequency activated dynamic. The correlation times of the fluctuations and their energy barriers are derived. The motion is favored at large Rh content, while it is hindered by the application of a magnetic field perpendicular to the FeAs layers. The same dynamic is observed in the spin-lattice relaxation rate, in a quantitatively consistent manner. These results are discussed in the light of nematic fluctuations involving domain wall motion. The analogies with the behaviour observed in the cuprates are also outlined.
\end{abstract}

PACS numbers: 74.70.Xa, 75.25.nj, 74.20.Mn

The study of the excitations in the normal phase of superconductors ( $\mathrm{SC}$ ) is of major importance to unravel the mechanisms driving the Cooper pair formation. Both in the cuprates and in the iron pnictides the presence of competing interactions gives rise to complex phase diagrams and to quasi-degenerate ground-states, which can induce unconventional dynamics at low energies. $\mathrm{Nu}-$ clear Magnetic Resonance (NMR) has played a key role in the study of the low-frequency (LF) excitations in the normal state both of high $\mathrm{T}_{c} \mathrm{SC}$ and, more recently, of the iron-based SC. Most of the NMR investigations carried out so far in these materials have concentrated on the dynamical features emerging from the spin-lattice re-

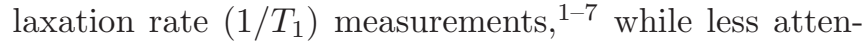
tion has been paid to the study of the spin-echo decay rate $1 / T_{2}$, which is quite a useful tool to probe very $L F$ excitations $\stackrel{-10}{-10}$ In the cuprates, one of the most significant achievements was the derivation of the staggered static spin susceptibility from ${ }^{63} \mathrm{Cu}(2)$ Gaussian echo decay rate $1 / T_{2 G}: \stackrel{11,12}{=}$ However other nuclei show a different behavior of $1 / T_{2}:{ }^{89} \mathrm{Y}$ NMR decay rate, in the $\mathrm{SC}$ phase of $\mathrm{YBa}_{2} \mathrm{Cu}_{3} \mathrm{O}_{7}$, presents an exponential term,,$\underline{13,14}$ which reveals a peak in $1 / T_{2}$, that was firstly ascribed

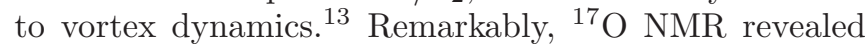
a second peak that, however, was also seen in ${ }^{63} \mathrm{Cu}$ NQR experiments in $\mathrm{YBa}_{2} \mathrm{Cu}_{3} \mathrm{O}_{7-x}$, where no magnetic field was applied, thus questioning the former explanation and suggesting other mechanisms involving charge fluctuations $\stackrel{14-16}{-16}$

Similar trends of $1 / T_{2}$ have been reported also in the recently discovered iron-based SC. In the optimally doped $\mathrm{Ba}\left(\mathrm{Fe}_{1-x} \mathrm{Co}_{x}\right)_{2} \mathrm{As}_{2}{ }^{9}$ and in $\mathrm{Ba}\left(\mathrm{Fe}_{1-x} \mathrm{Rh}_{x}\right)_{2} \mathrm{As}_{2}$ (BaFeRh122 hereafter), $\stackrel{8}{=}$ a peak in $1 / T_{2}$ was detected below $T_{c}$ and again associated with the vortex dynamics. On the other hand, the behavior of ${ }^{75} \mathrm{As}$ NMR $T_{2}$ in the normal phase of BaFeRh122 SC is not completely understood $\$ .99$ and its magnitude is far from any theoretical expectation.
In the following, a systematic study of ${ }^{75} \mathrm{As}$ NMR spin echo decay in BaFeRh122 iron-based SC, over a broad range of $\mathrm{Rh}$ doping, is presented. The echo shows a high temperature (T) dominant Gaussian decay which becomes exponential at low $\mathrm{T}$. The exponential decay rate increases upon cooling already in the normal phase, suggesting the onset of an unconventional very LF activated dynamics, whose characteristic correlation times are derived together with the corresponding energy barriers. This dynamic persists across the whole phase diagram up to the overdoped compounds, but it is less pronounced if the magnetic field is applied perpendicularly to the FeAs layers. It is also shown that the same dynamics affect $1 / T_{1}$. These LF fluctuations are discussed in terms of domain wall motion, possibly involving nematic fluctuations.

NMR measurements have been performed on three BaFeRh122 single crystals: an underdoped sample, with $\mathrm{x}=4.1 \%\left(T_{c}=13.6 \mathrm{~K}\right)$, a nearly optimally doped sample, with $\mathrm{x}=6.8 \%\left(T_{c}=22.4 \mathrm{~K}\right)$, and an overdoped sample, with $\mathrm{x}=9.4 \%\left(T_{c}=15.1 \mathrm{~K}\right)$. The samples were grown as outlined in Ref. 17. The critical temperature $T_{c}$ was determined via Superconductor Quantum Interference Device (SQUID) magnetometry and it is in agreement with Ref. 17. ${ }^{75} \mathrm{As}$ NMR experiments were performed at $6.4 \mathrm{~T}, 9 \mathrm{~T}$ and $11 \mathrm{~T}$, for $\mathbf{H}_{\mathbf{0}}$ parallel and perpendicular to the $c$ axis. The spin echo decay time was estimated by fitting the decay of the transverse nuclear magnetization $M_{t}$, measured either after a standard Hahn echo (HE) sequence $(\pi / 2-\tau-\pi)$ or by using a Carr-Purcell-Meiboom-Gill ( $C P M G$ ) sequence $\left(\pi / 2_{x}-\tau_{C P}-\pi_{y}-\tau_{C P}-\pi_{y} \ldots\right)$. In the latter, the delay $\tau_{C P}$ was varied in order to extract the intrinsic decay time $T_{2 C P M G}$ for $\tau_{C P} \rightarrow 0$. The $H E$ decay was first corrected in order to remove the contribution of the spinlattice relaxation rate $1 / T_{1} \underline{18}$ Afterwards, the echo decay could be nicely fit by the product of an exponential and 
a Gaussian decay (inset of Fig. 1):

$$
M_{t}(2 \tau) / M_{0}=\exp \left(-2 \tau / T_{2 \exp }\right) \exp \left(-(2 \tau)^{2} / 2 T_{2 G}^{2}\right) .
$$

The two components evolve with temperature in such a way that, in the high $\mathrm{T}$ regime, the Gaussian term is significantly larger than the exponential one, and both are weakly temperature dependent. This trend persists down to a temperature $T^{*}>T_{c}$, where the Gaussian contribution becomes negligible, while the exponential rate grows rapidly and becomes the main contribution to the echo decay (Figs. 1 and 2). The experimental values of $T^{*}$ are $22 \pm 2 \mathrm{~K}$ and $18 \pm 2 \mathrm{~K}$ for $x=6.8 \%$ and $x=9.4 \%$, respectively. Here the low T exponential component is discussed, since the high $\mathrm{T}$ constant behavior has been discussed by $\mathrm{Oh}$ et al. $\stackrel{9}{ }$ From Fig 3 one notices that also $1 / T_{2 C P M G}$ is significantly reduced with respect to $1 / T_{2 \exp }$ for $T<T^{*}$ and shows a less pronounced field and $\mathrm{T}$ dependence. The $H E$ and $C P M G$ sequences are most sensitive to fluctuations with a characteristic time scale $\tau_{c} \sim \tau$ and $\sim \tau_{C P}$, respectively. Hence, the observed difference between $1 / T_{2 C P M G}$ and $1 / T_{\text {exp }}$ suggests that the correlation time of the fluctuations probed by ${ }^{75}$ As nuclei increases above the $\mu s$, below $T^{*}$. By comparing the measurements performed on the three crystals, for different magnetic field orientations (Fig. 2) and magnitudes (Figs. 2]and 3), one can conclude that the enhancement of $1 / T_{2 \exp }$ has four main features: (i) it starts above $T_{c}$, (ii) it is favored by the magnetic field, (iii) it is accentuated for in-plane fields and (iv) it persists across the whole phase diagram, up to the overdoped compound (Fig. 3).

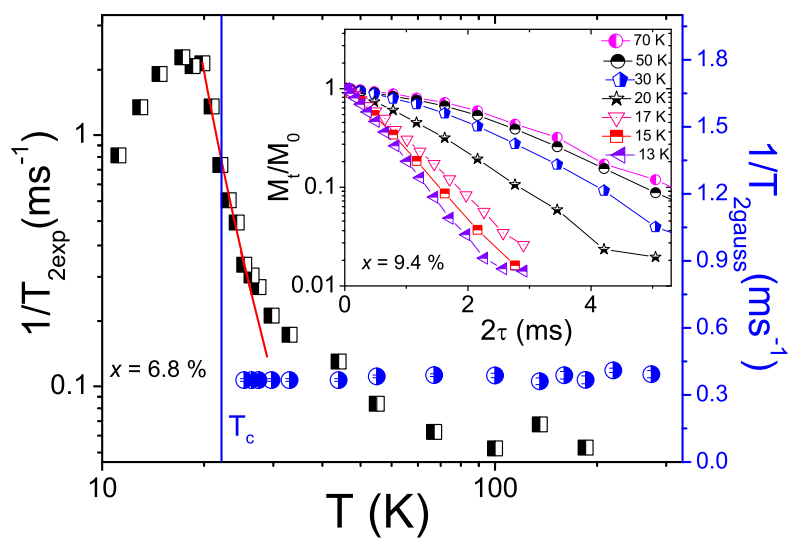

FIG. 1: The exponential (black half filled squares) and Gaussian (blue half filled circles) $1 / T_{2}$ measured by Hahn echo at $11 \mathrm{~T} \| c$ of the $x=6.8 \%$ sample. The red solid line shows the best fit to fast motion equation (see text). (Inset) The spin-echo amplitude decay at different $\mathrm{T}$, for $x=9.4 \%$, at $\mathbf{H}_{0}=11 \mathrm{~T} \| c$ axis, corrected by the $T_{1}$ contribution.

Further insights into the LF dynamics can be derived from the T-dependence of the full width at half maximum (FWHM) of the central $1 / 2 \rightarrow-1 / 2$ NMR line, obtained from the Fourier transform of half of the echo
(Fig. 2). The NMR spectrum displays an inhomogeneously broad lineshape with a LF tail, and it gets broader upon cooling. Remarkably, at the same temperature $T^{*}$ where the echo decay becomes exponential, the linewidth starts to decrease, hence suggesting the onset of LF dynamics which can average-out the static frequency distribution probed by ${ }^{75}$ As nuclei. Finally, at $T_{m}<T_{c}$, the line broadens again (Fig. 2), as expected when the solid/glassy vortex phase sets in $\underline{\underline{8}, 9,19}$

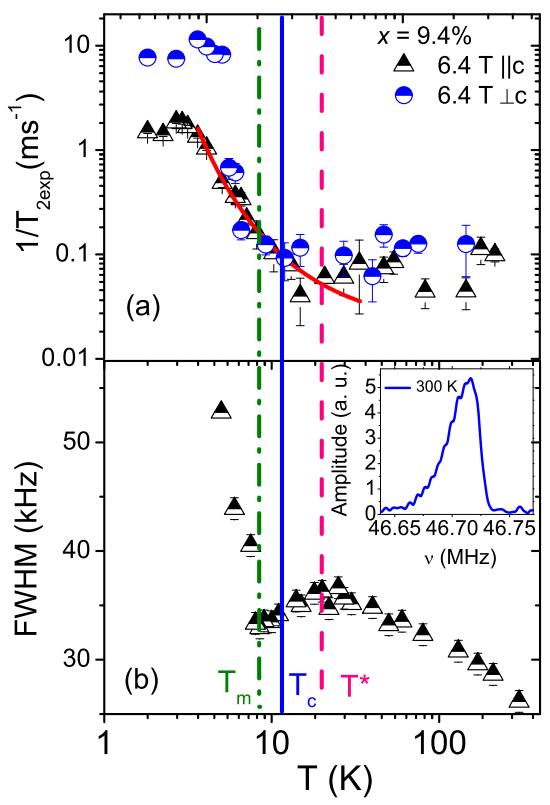

FIG. 2: (a) $1 / T_{2 \exp }$ measured at $H_{0}=6.4 \mathrm{~T} \| c$, for $x=9.4 \%$. The red solid line shows the best fit according to the fast motion equation (see text). The blue circles show $1 / T_{2 \exp }$ measured at $H_{0}=6.4 \mathrm{~T} \perp c$. (b) The $\mathrm{T}$ dependence of the FWHM of ${ }^{75}$ As central line: the width increases with decreasing $\mathrm{T}$ down to $T^{*}$ (red dashed line), where it starts to decrease. Finally the linewidth increases again at the vortex freezing temperature $T_{m}$ (green dashed-dotted line). The blue line marks $T_{c}$ at $6.4 \mathrm{~T}$. The inset displays an example of the ${ }^{75}$ As NMR spectrum, showing a clear asymmetry.

Since a LF dynamic is present one should expect an effect also on $1 / T_{1}$, which probes the spectral density at the nuclear Larmor frequency. In the inset of Fig. 3 it is shown $1 / T_{1}$, as derived after a saturation recovery pulse sequence, for both magnetic field orientations, for $x=9.4 \%$. Remarkably, a bump in the spin-lattice relaxation rate was observed in the normal state, when $\mathbf{H}_{\mathbf{0}} \perp \mathrm{c}$, which is nearly absent for $\mathbf{H}_{\mathbf{0}} \|$ c. Notice that the corresponding $1 / T_{1} T$ data for $\mathbf{H}_{0} \perp \mathrm{c}$ are quantitatively in agreement to those measured by Ning et $a l^{\underline{4}}$ in $\mathrm{Ba}\left(\mathrm{Fe}_{1-x} \mathrm{Co}_{x}\right)_{2} \mathrm{As}_{2}$ crystals for the same magnetic field orientation. The peak behavior of $1 / T_{1}$ for the two orientations is suggestive of the Bloembergen-Purcell-Pound

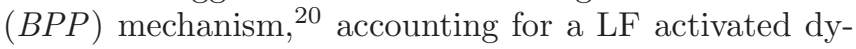
namic (Fig. 4), plus a power law trend $T^{\alpha}(\alpha \rightarrow 1)$, which characterizes these compounds when $\mathbf{H}_{\mathbf{0}} \|$ c. A fit to this model results into an energy barrier $U=50 \pm 5$ 
$\mathrm{K}$ corresponding to a correlation time at infinite temperature $\tau_{0}=5.2 \times 10^{-10} s$ and an average fluctuating field $h_{e \perp}=19.4 \pm 2.2 \mathrm{G}$. Such parameters are comparable with the ones recently reported by Hammerath et al. in underdoped $\mathrm{LaO}_{1-x} \mathrm{~F}_{x} \mathrm{FeAs}{ }^{21}$ In principle, two possible reasons for the anisotropy in $1 / T_{1}$ should be considered: (i) the role of the magnetic field in inhibiting the LF fluctuations when $\mathbf{H}_{\mathbf{0}} \| \mathrm{c}$, (ii) the filter effect of the hyperfine form factor $\stackrel{22}{2}$ Given the behaviour found for $1 / T_{2 \exp }$ for the different magnetic field orientations (see Fig. 2a) the former scenario appears more likely.

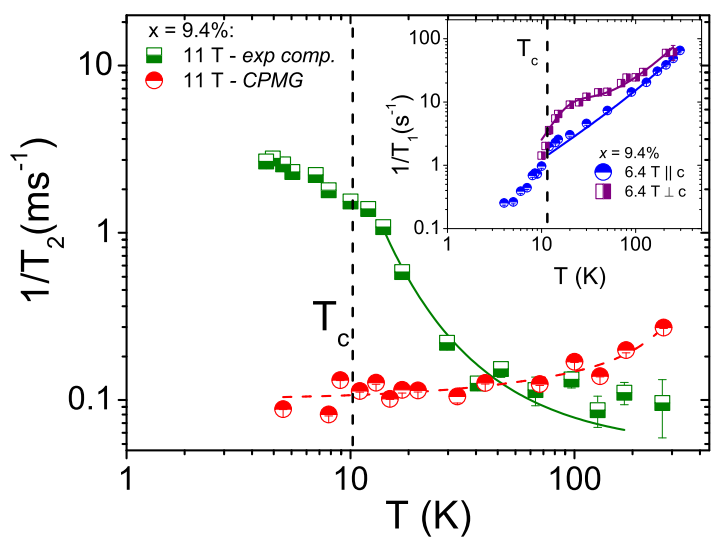

FIG. 3: The $H E 1 / T_{2 \exp }$ is shown for the overdoped sample, at $11 \mathrm{~T} \| c$ (green half filled squares). The red half filled circles show the $1 / T_{2 C P M G}$ at $11 \mathrm{~T} \| c$. The green solid line is the best fit according fast motion equation, while the dashed line is a guide-to-the-eye. The dashed vertical line indicates $T_{c}$. The inset displays $1 / T_{1}$ data collected at $6.4 \mathrm{~T} \|$ (blue circles) and $\perp$ (purple squares) to the $c$ axis. The solid lines are $1 / T_{1}$ best fits according to a power law (blue line) or to the sum of a BPP and a power law (purple line).

Further quantitative information on the correlation times describing the LF activated dynamics can be gained from the analysis of the spin echo decay rate $1 / T_{2 \exp }$. When the $H E$ becomes exponential, the spinecho relaxation rate can be fit by the fast motions expression $\underline{23}$

$$
1 / T_{2 \exp }={ }^{75} \gamma^{2}<h_{e \|}^{2}>\tau_{0} e^{U(H, x) / T},
$$

where the energy barrier $U$ is assumed to depend on the field intensity $H_{0}$ and on the Rh concentration $x$. The fit results, obtained by using the $\tau_{0}$ value derived from $1 / T_{1}$,

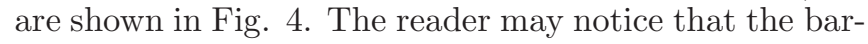
rier is comparable with the one found from the $1 / T_{1}$ fit. Moreover $U$ is $H_{0}$-independent, in the explored range, while it clearly depends on the electronic concentration, namely it decreases by increasing the Rh content. This trend indicates that the higher the electron doping the faster the dynamics. Furthermore the fluctuating longitudinal local field $h_{e \|}$ shows a continuous increase with the applied field and the doping. It is also noticed that, when the field is perpendicular to the $c$ axis, the enhancement is significantly larger.

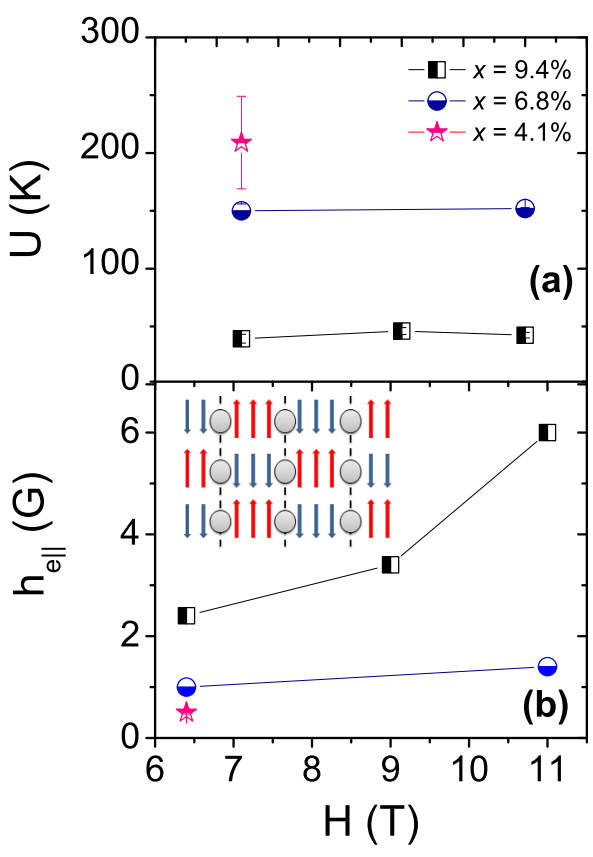

FIG. 4: (a) Energy barriers estimated from $1 / T_{2 \exp }$ according to fast motion equation at different doping levels. (b) The field dependence of the amplitude of the longitudinal field fluctuations at different Rh contents. The inset shows a sketch where columnar antiferromagnetic regions are separated by antiphase domain wall: the blue/red arrows stand respectively for the down/up spins, while the grey circles indicate the electronic charges which may favor the domain formation.

The LF dynamics evidenced by $1 / T_{2 \exp }$ and by the bump in $1 / T_{1}$ cannot be due to the standard correlated electron spin fluctuations or SC fluctuations which typically occur at frequencies orders of magnitude larger than the frequency probed here. One should look for very LF fluctuations as the ones occurring close to a spin or charge freezing or taking place among quasi-degenerate groundstates. In this respect, one should consider that, owing to the geometry of the relevant exchange couplings, the magnetic properties of the iron pnictides have often been described within an effective $\mathrm{J}_{1}-\mathrm{J}_{2}$ model on a square lattice $\stackrel{24}{\underline{w}}$ The ground-state of that model is characterized by two degenerate columnar antiferromagnetic ground-states corresponding to two nematic phases, and the fluctuations between those two states can give rise to very $L F$ dynamics. In fact in vanadates, which can be considered as prototypes of that model, these LF fluctuations have been detected by $\mu \mathrm{SR}$ above the magnetic ordering, 25 in a temperature range where the electron spins are already correlated $\stackrel{26}{=}$ These dynamics can be associated with domain wall motion separating correlated regions of the two nematic states. Once the two different phases set in, the domain walls can be put into motion if the energy barrier $U$ separating these two phases 27 is overcome (Fig. 4). Notice that a similar scenario has also been proposed in a recent study of the magnetic state of $\mathrm{CaFe}_{2} \mathrm{As}_{2} \stackrel{10}{\underline{10}}$ 
On the other hand, the observations of the magnetic field effect on $1 / T_{2}$ and $1 / T_{1}$, suggest that when $\mathbf{H}_{\mathbf{0}} \| \mathrm{c}$ the fluctuations are reduced. Such an effect recalls the fieldinduced charge order recently reported in underdoped $\mathrm{YBa}_{2} \mathrm{Cu}_{3} \mathrm{O}_{6+x} \mathrm{SC}$, where the blocking of the charge order occurs only if $\mathbf{H}_{\mathbf{0}}$ is perpendicular to the highly conductive $\mathrm{CuO}_{2}$ layers ${ }^{28,29}$ Moreover in these systems, Wu et $a l_{1}^{28}$ found a temperature and magnetic field dependence of $1 / T_{2}$ similar to the one reported here for the iron-based SC. In the light of these analogies one could speculate that very LF fluctuations associated with domain wall motion, possibly involving charge stripes, can be present both in the cuprates and in the iron-based SC. However, further experiments are required to support such a scenario.

In conclusion, this paper presents a systematic study of the spin-echo decay rate in 122 iron-pnictides over a broad doping range. A LF spin dynamic is observed above $T_{c}$ and it is responsible for a bump in the spinlattice relaxation time, as well as for an enhancement of the exponential component of the spin-echo decay rate.
Such dynamics are common at all the doping concentrations, and they get faster in the overdoped regime. Moreover they can be associated with domain wall motion, possibly involving nematic fluctuations. The remarkable analogies with the behavior found in the cuprates indicate the need for a deeper investigation, also by other techniques.

We would like to thank A. Mounce, J. P. Lee, F. Hammerath and S.-J. Yuan for help and useful discussion. The research activity in Pavia was supported by Fondazione Cariplo (Research Grant No. 2011-0266). Research at N. U. was supported by the U.S. DOE, Office of Basic Energy Sciences (BES), Division of Materials Sciences and Engineering (MSE), award DE-FG0205ER46248. Work done in Ames Laboratory (P.C.C.) was supported by the U.S. DOE, BES Office, MSE Division under contract No. DE-AC02-07CH11358. A portion of this work was performed at the NHMFL, which is supported by National Science Foundation Cooperative Agreement No. DMR-0654118, the State of Florida, and the U.S. DOE.
1 K. Kitagawa, N. Katayama, K. Ohgushi, M. Yoshida and M. Takigawa, JPSJ 77, 114709 (2008).

${ }^{2}$ K. Kitagawa, Y. Nakai, T. Iye, K. Ishida, Y. Kamihara, M. Hirano and H. Hosono, Phys. Rev. B 81, 212502 (2010).

3 M. Fu, D. A. Torchetti, T. Imai, F.L. Ning, J.-Q. Yan, and A. S. Sefat, Phys. Rev. Lett. 109, 247001 (2012).

4 F. L. Ning, K. Ahilan, T. Imai, A. S. Sefat, M. A. McGuire, B. C. Sales, D. Mandrus, P. Cheng, B. Shen, and H.-H Wen, Phys. Rev. Lett. 104, 037001 (2010).

${ }^{5}$ D. A. Torchetti, M. Fu, D. C. Christensen, K. J. Nelson, T. Imai, H. C. Lei, and C. Petrovic, Phys. Rev. B 83, 104508 (2011)

6 D. Parker, O. V. Dolgov, M. M. Korshunov, A. A. Golubov, and I. I. Mazin, Phys. Rev. B 78, 134524 (2008).

7 S. Oh, A. M. Mounce, W. P. Halperin, C. L. Zhang, P. Dai, A. P. Reyes, and P. L. Kuhns, Phys. Rev. B 85, 174508 (2012).

8 L. Bossoni, P. Carretta, A. Thaler and P. C. Canfield, Phys. Rev. B 85, 104525 (2012).

9 S. Oh, A. M. Mounce, S. Mukhopadhyay, W. P. Halperin, A. B. Vorontsov, S. L. Bud'ko, P. C. Canfield, Y. Furukawa, A. P. Reyes, and P. L. Kuhns, Phys. Rev. B 83, 214501 (2011).

10 H. Xiao, T. Hu, A. P. Dioguardi, N. Roberts-Warren, A. C. Shockley, J. Crocker, D. M. Nisson, Z. Viskadourakis, Xianyang Tee, I. Radulov, C. C. Almasan, N. J. Curro, and C. Panagopoulos, Phys. Rev. B 85, 024530 (2012).

11 M. Takigawa, Phys. Rev. B 49, 4158 (1994).

12 C. H. Pennington and C. P. Slichter, Phys. Rev. Lett. 66, 381 (1991).

13 B. J. Suh, D. R. Torgeson and F. Borsa, Phys. Rev. Lett. 71, 3543 (1993). B. J. Suh. PhD thesis.

14 C. H. Recchia, J. A. Martindale, C. H. Pennington, W. L. Hults and J. L. Smith, Phys. Rev. Lett. 78, 3543 (1997).

15 S. Krämer and M. Mehring, Phys. Rev. Lett. 83, 396 (1999).
16 A. V. Bondar et al., JETP Lett. 50, 146 (1989). K. Kumagai et al., JPSJ 59, 2336 (1990). Y. Itoh et al., JPSJ 59, 3463 (1990).

17 N. Ni, M. E. Tillman, J.-Q. Yan, A. Kracher, S. T. Hannahs, S. L. Bud'ko and P. C. Canfield, Phys. Rev. B 78 214515 (2008). N. Ni, A. Thaler, A. Kracher, J.-Q. Yan, S. L. Bud'ko and P. C. Canfield, Phys. Rev. B 80024511 (2009).

18 R. E. Walstedt and S.-W. Cheong, Phys. Rev. B 51, 3163 (1995).

19 L. Bossoni, P. Carretta, M. Horvatić, M. Corti, A. Thaler and P. C. Canfield, Eur. Phys. Lett. 102, 17005 (2013).

20 A. Abragam, Principles of Nuclear Magnetism (Oxford University Press) New York (1961).

21 F. Hammerath, U. Gräfe, H. Kühne, P. L. Kuhns, A. P. Reyes, G. Lang, S. Wurmehl, B. Büchner, P. Carretta, and H.-J. Grafe, http://arxiv.org/abs/1307.3138v1 (2013).

22 A. Smerald and N. Shannon, Phys. Rev. B 84, 184437 (2011).

23 M. Takigawa and G. Saito, JPSJ 55, 1233 (1986).

${ }^{24}$ Q. Si and E. Abrahams, Phys. Rev. Lett. 101, 076401 (2008). C. de la Cruz et al., Nature (London) 453, 899 (2008). P. Dai, Jiangping $\mathrm{Hu}$, and Elbio Dagotto, Nat. Phys. 8, 709 (2012).

25 P. Carretta, R. Melzi, N. Papinutto and P. Millet, Phys. Rev. Lett. 88, 047601 (2002).

26 L. Bossoni, P. Carretta, R. Nath, M. Moscardini, M. Baenitz, and C. Geibel, Phys. Rev. B 83, 014412 (2011).

27 P. Chandra, P. Coleman, and A. I. Larkin, Phys. Rev. Lett. 64, 88 (1990).

28 T. Wu, H. Mayaffre, S. Krämer, M. Horvatić, C. Berthier, W. N. Hardy, R. Liang, D. A. Bonn and M.-H. Julien, Nature 477, 191 (2011). J. M. Tranquada, B. J. Sternlieb, J. D. Axe, Y. Nakamura, and S. Uchida, Nature 375, 561 (1995).

29 T. Wu, H. Mayaffre, S. Kraemer, M. Horvatić, C. Berthier, 
C.T. Lin, D. Haug, T. Loew, V. Hinkov, B. Keimer, M.-H. Julien, http://arxiv.org/abs/1306.6306v1 (2013). 